\title{
2082 "Two-dimensional M-Mode" display of the mitral valve from CMR cine acquisitions: insights into normal leaflet and annular motion

\author{
John KM Chan*1, Robert Merrifield ${ }^{2}$, Ricardo R Wage ${ }^{1}$, Karen Symmonds ${ }^{1}$, \\ Tim Cannell ${ }^{1}$, David N Firmin ${ }^{1}$, John R Pepper ${ }^{1}$, Dudley J Pennell ${ }^{1}$ and \\ Philip J Kilner ${ }^{1}$
}

Address: ${ }^{1}$ Royal Brompton Hospital, London, UK and ${ }^{2}$ Imperial College, London, UK

* Corresponding author

from I th Annual SCMR Scientific Sessions

Los Angeles, CA, USA. I-3 February 2008

Published: 22 October 2008

Journal of Cardiovascular Magnetic Resonance 2008, I0(SuppI I):A35I doi:I0.I I86/I532-429X-I0-SI-A35 I

This abstract is available from: http://jcmr-online.com/content/I0/SI/A35 I

(c) 2008 Chan et al; licensee BioMed Central Ltd.

\begin{abstract}
Introduction
CMR assessment of the mitral valve provides unique insights into its normal annular and leaflet motion. Although various other modalities have investigated mitral annular and leaflet motion in isolation, CMR imaging allows assessment of both the temporal and spatial relationship of mitral annular and leaflet motion throughout the cardiac cycle. Such insights provided by CMR may be important in understanding various disease processes affecting the mitral valve and the impact of these on normal mitral valve function, and in developing techniques for the surgical repair of diseased mitral valves.
\end{abstract}

\section{Methods}

Eight healthy volunteers (all males, aged 32-51 years; mean age $37.9+/-6.8$ years) with no known history of cardiac disease were studied. CMR imaging was performed on a $1.5 \mathrm{~T}$ scanner (Siemens Avanto). SSFP end-expiratory breath hold cines were acquired in vertical and horizontal long axis planes and contiguous short axis cines from the atrial-ventricular junction to the apex of the ventricle. Retrospective ECG triggering for capture of the entire cardiac cycle was used. Images were acquired at high temporal resolution, repetition time $1.86 \mathrm{~ms}$, echo time $1.13 \mathrm{~ms}$, in plane pixel size $1.7 \times 1.7 \mathrm{~mm}$, flip angle 80 degrees, acquisition time 16 heart beats, slice thickness $7 \mathrm{~mm}$. Measurements and analysis of the mitral annular and leaflet motion were made from the long axis LVOT view from which the septal-lateral diameter of the mitral annulus was also determined. The commissure-commissure mitral annular diameter was determined from an oblique long axis cine aligned through both commissures. The motions of the mitral valve annulus and leaflets were then traced in 2-dimensions from individual cine frames (Fig 1) and displayed graphically against time ( $\mathrm{x}$ axis) to demonstrate both their spatial and temporal relationships (Fig 2).

\section{Results}

Six phases of mitral annular and leaflet motion were identified during the cardiac cycle:

\section{(I) Ventricular systolic excursion}

The excursion of the mitral annulus towards the LV apex was asymmetrical with greater excursion of the posterior annulus and leaflets $(13.5 \mathrm{~mm})$ compared to the anterior annulus and leaflets $(12.0 \mathrm{~mm})$. The septal-lateral diameter increased in size during this phase $(27.0 \mathrm{~mm}$ to 31.4 $\mathrm{mm}$ ) while the commissure-commissure diameter remained unchanged. Mean phase duration: $376 \mathrm{~ms}$ ( $42 \%$ of cardiac cycle).

\section{(II) Leaflet opening and annular recoil}

Mitral leaflet opening was followed closely by atrially directed recoil of the mitral annulus. Separation of the 

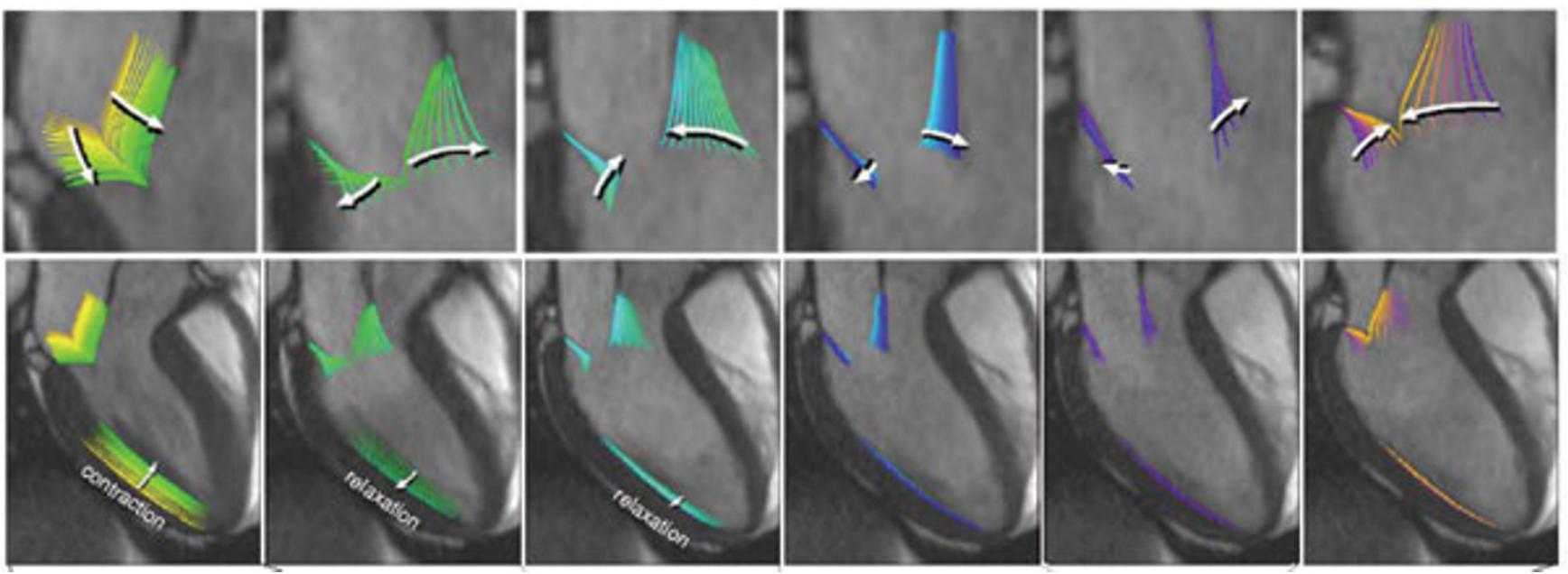

\section{Figure I}

The six phase of mitral annular and leaflet motion during the cardiac cycle. Our study of 2D M-Mode display of the normal mitral valve has identified six phases of the cardiac cycle. This has provided important insights into normal leaflet and annular motion.

mitral leaflet edges was greatest in the middle of this phase. Mean phase duration: $164 \mathrm{~ms}$ (18\% of cardiac cycle).

\section{(III) Leaflet approximation}

The mitral leaflets move to a partially closed position. Mean phase duration: $94 \mathrm{~ms}$ (10\% of cardiac cycle).

\section{(IV) Mid diastolic pause}

This represents the phase of diastolic inactivity with the mitral leaflets drifting in a neutral position. Mean phase duration: $146 \mathrm{~ms}$ ( $14 \%$ of cardiac cycle).

\section{(V) Atrial systolic excursion}

Left atrial systole pulls the mitral annulus and impells more blood into the ventricle which increases the separation of the mitral leaflet edges. Mean phase duration: 131 ms ( $14 \%$ of cardiac cycle).

\section{Conclusion}

CMR assessment and graphic 2-D display of the mitral valve gives new insights into the dynamic nature of mitral annular and leaflet motions through the cardiac cycle. This may be important in the understanding of disease processes affecting the mitral valve and its impact on mitral valve function, and in the design of flexible mitral annuloplasty rings or bands which would allow preservation of the normal dynamic mitral annular and leaflet motion following surgical repair.

\section{(VI) Leaflet closure}

This marks the onset of systole.

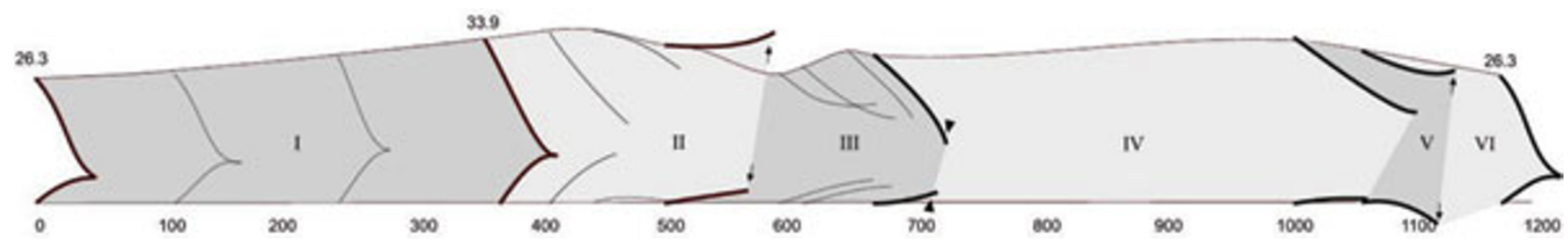

\section{Figure 2}

Two dimensional M-Mode display of mitral annular and leaflet motion during the cardiac cycle in a healthy volunteer. Arrows indicate maximal early and late diastolicopening of the valve tips and arrow heads the diastolic leaflet approximation. 